\title{
Lipid peroxidation, detoxification capacity, and genome damage in mice after transplacental exposure to pharmaceutical drugs
}

\author{
D. Markovic ${ }^{1}$, J. Katic ${ }^{2}$, R. Stojkovic ${ }^{3}$, S. Borovic ${ }^{3}$, N. Zarkovic $^{3}$ and A. Fucic ${ }^{2}$ \\ ${ }^{1}$ Galapagos Research Center, Zagreb, Croatia \\ ${ }^{2}$ Institute for Medical Research and Occupational Health, Zagreb, Croatia \\ ${ }^{3}$ Rudjer Boskovic Institute, Zagreb, Croatia
}

\begin{abstract}
Data on genome damage, lipid peroxidation, and levels of glutathione peroxidase (GPX) in newborns after transplacental exposure to xenobiotics are rare and insufficient for risk assessment. The aim of the current study was to analyze, in an animal model, transplacental genotoxicity, lipid peroxidation, and detoxification disturbances caused by the following drugs commonly prescribed to pregnant women: paracetamol, fluconazole, 5-nitrofurantoin, and sodium valproate. Genome damage in dams and their newborn pups transplacentally exposed to these drugs was investigated using the in vivo micronucleus (MN) assay. The drugs were administered to dams intraperitoneally in three consecutive daily doses between days 12 and 14 of pregnancy. The results were correlated, with detoxification capacity of the newborn pups measured by the levels of GPX in blood and lipid peroxidation in liver measured by malondialdehyde (HPLC-MDA) levels. Sodium valproate and 5-nitrofurantoin significantly increased MN frequency in pregnant dams. A significant increase in the MN frequency of newborn pups was detected for all drugs tested. This paper also provides reference levels of MDA in newborn pups, according to which all drugs tested significantly lowered MDA levels of newborn pups, while blood GPX activity dropped significantly only after exposure to paracetamol. The GPX reduction reflected systemic oxidative stress, which is known to occur with paracetamol treatment. The reduction of MDA in the liver is suggested to be an unspecific metabolic reaction to the drugs that express cytotoxic, in particular hepatotoxic, effects associated with oxidative stress and lipid peroxidation.
\end{abstract}

Key words: Glutathione peroxidase; Malondialdehyde; Genome damage; Transplacental exposure

\section{Introduction}

Limited data are available on the mechanisms of transplacental genotoxicity and detoxification in the fetus during intrauterine development. Transplacental exposure to xenobiotics may disturb developmental homeostasis and cause physiological stress and genome damage in the fetus, with a lifelong increase in health risks for different diseases (1). In addition to environmental exposure and diet, drug intake during pregnancy could have a significant impact on the genomic and nongenomic pathways of fetal development. Although the teratogenic effect of many drugs has been described, the mechanisms and contribution to genome damage etiology have not been elucidated.

During pregnancy the metabolism in women changes (2), as reflected in specific biological effects of drugs and environmental stressors. Drug metabolism may produce reactive oxygen species, which cause oxidative damage to DNA, RNA, lipids, and proteins via superoxide anion, hydrogen peroxide, and hydroxyl radicals (3). Lipid peroxidation is a process also triggered by free radicals, which results in the degradation of polyunsaturated fatty acids (4). The lipid peroxidation end-products malondialdehyde (MDA) and 4-hydroxy-2-nonenal (HNE) adversely affect genome integrity, causing DNA strand breaks, formation of DNA adducts, increased frequency of sister chromatid exchange, and apoptosis $(5,6)$.

Drugs and/or their metabolites can accumulate in the tissues of the fetus in a different way than in adults, because of the specific metabolism and high tissue proliferation rate in the fetus (7). The dynamic balance 
between Phase I and II drug-metabolizing enzymes influences the toxic effects of agents metabolized at different stages of development and consequently the timing and level of DNA damage (8). The levels of glutathione-related antioxidant enzymes differ significantly in fetal and adult rat liver, with very low activity during the perinatal period (9).

Differences in enzymatic systems, pharmacokinetics, and clearance capacity between the fetus and adult render simple extrapolation of results obtained in adult organisms prone to errors when assessing fetal health risk after exposure to xenobiotics (10).

The animal model of transplacental exposure can significantly elucidate involved mechanisms and give insight into the health risks associated with drugs whose metabolites and doses are known from clinical practice. Such results can also contribute to the investigation of health effects after transplacental exposure to environmental xenobiotics.

In animal studies on pregnant dams, paracetamol has been shown to cause liver and kidney ultrastructural changes after long-term treatment (11), whereas 5-nitrofurantoin has also been shown to be genotoxic (12) and is associated with birth defects (13). The management of epilepsy in pregnant women requires careful monitoring, because antiepileptic drugs such as sodium valproate are related to teratogenicity and oxidative stress and a decrease in antioxidants such as vitamin $\mathrm{E}$ and glutathione peroxidase (GPX) $(14,15)$. Although no increased risk of malformations was found in a population-based study after treatment with the antifungal drug fluconazole (16), in animal models it has been shown to be transplacentally genotoxic and was associated with embriotoxic and teratogenic effects $(17,18)$.

The aim of the present study was to measure genome damage in newborn pups transplacentally exposed to selected drugs often used during pregnancy (19-22), using an in vivo micronucleus (MN) assay (23), and to compare the results with levels of GPX and MDA. The drugs used in the current study to treat the pregnant mice were analgesic/antipyretic (paracetamol), antifungal (fluconazole), antibiotic (5-nitrofurantoin), and antiepileptic (sodium valproate). Additionally, because antibiotics and paracetamol are frequently prescribed simultaneously, the combination of 5-nitrofurantoin and paracetamol was also investigated, in order to detect possible synergistic or antagonistic mechanisms in the case of combined exposure. It is known that paracetamol, fluconazole, sodium valproate, 5-nitrofurantoin, and cyclophosphamide (CP) cross the placenta (24-29). Applied doses of drugs were taken from maximal daily doses in human medical practice (30). Administration of the drugs to dams was performed for 3 days, from days 12 through 14 of pregnancy, in order to target the period of major organogenesis in mice (31).

\section{Material and Methods}

This study included 56 2-month-old BALB/c dams (Charles River, Germany), randomized into 7 groups, and their newborn pups. The mice were obtained from the breeding colony at the Rudjer Boskovic Institute (Zagreb, Croatia). Dams were kept with the litter in separate cages under the following conditions: temperature $22 \pm 2^{\circ} \mathrm{C}$, relative humidity $55 \pm 10 \%$, with about $15-20$ air changes per hour and a 12:12-h light/dark cycle. All animals had access to food and water ad libitum. All experiments were performed according to the ILAR Guide for the Care and Use of Laboratory Animals, Council Directive (86/609/ EEC) and the Croatian Animal Welfare Act (NN 19/99). This study was approved by the Croatian Ministry of Agriculture (Veterinary Department, Ethics Committee approval No. 525-06-05-02).

\section{Drug administration}

Five groups of pregnant dams received the following pharmaceutical products: 5-nitrofurantoin (Sigma, USA), fluconazole (Sigma), paracetamol (Belupo, Croatia), 5nitrofurantoin (Sigma), and sodium valproate (Krka, Slovenia). One group received a combination of 5nitrofurantoin and paracetamol. The positive control group received CP (Sigma), and the negative control group received physiological saline (Kemika, Croatia). There were eight dams in each group.

The administered volume for each compound was $5 \mathrm{~mL} / \mathrm{kg}$. The drug doses (Table 1) were based on recommended human doses (30). Preliminary studies had shown that the positive control of $10 \mathrm{mg} / \mathrm{kg} \mathrm{CP}$ was suitable for studying transplacental genotoxicity, as it shows a significant increase in $\mathrm{MN}$ frequency but not significant fetotoxicity. All drugs were administered intraperitoneally in three consecutive daily doses on days 12 to 14 of pregnancy, except CP, which was administrated in a single dose. Samples were taken from the dams $48 \mathrm{~h}$ after drug administration for in vivo $\mathrm{MN}$ assays.

In each litter, 6 animals were analyzed using an in vivo MN assay, GPX activity, and MDA analysis. Blood samples were taken for the in vivo $\mathrm{MN}$ assay and glutathione, and liver samples were taken for MDA measurement. Dams and newborn pups were killed after delivery.

\section{In vivo $\mathrm{MN}$ testing}

Peripheral blood ( $5 \mu \mathrm{L}$ ) was collected from the tail vein of pregnant dams on day 12 of pregnancy and $48 \mathrm{~h}$ after drug administration. Neonatal genotoxicity in pups was analyzed from 6 to $18 \mathrm{~h}$ after delivery. Blood was smeared on an acridine orange-coated slide (Molecular Probes, USA), covered with a cover slip, and analyzed according to Hayashi et al. (23). For each animal, 1000 reticulocytes were analyzed using a fluorescent microscope 
Table 1. Concentration of drugs used in the study.

\begin{tabular}{lllc}
\hline Group & \multicolumn{1}{c}{ Drug (solvent) } & Molecular formula & Dose (mg/kg) \\
\hline 1 & Physiological saline & $\mathrm{NaCl}$ & 10 (single) \\
2 & Cyclophosphamide (saline) & $\mathrm{C}_{7} \mathrm{H}_{15} \mathrm{Cl}_{2} \mathrm{~N}_{2} \mathrm{O}_{2} \mathrm{P}$ & 50 \\
3 & 5-Nitrofurantoin (dimethyl cellulose) & $\mathrm{C}_{8} \mathrm{H}_{6} \mathrm{~N}_{4} \mathrm{O}_{5}$ & 12 \\
4 & Fluconazole (saline) & $\mathrm{C}_{13} \mathrm{H}_{12} \mathrm{~F}_{2} \mathrm{~N}_{6} \mathrm{O}$ & 60 \\
5 & Paracetamol (saline) & $\mathrm{C}_{8} \mathrm{H}_{9} \mathrm{NO}_{2}$ & $50 / 60$ \\
6 & 5-Nitrofurantoin (dimethyl cellulose)/paracetamol & $\mathrm{C}_{8} \mathrm{H}_{6} \mathrm{~N}_{4} \mathrm{O}_{5} / \mathrm{C}_{8} \mathrm{H}_{9} \mathrm{NO}_{2}$ & 100 \\
7 & (saline) & & $\mathrm{C}_{8} \mathrm{H}_{15} \mathrm{NaO}_{2}$ \\
\hline
\end{tabular}

(Olympus, Japan).

\section{Determination of GPX activity}

Blood GPX activity was analyzed from whole blood with a UV method using the commercial Ransel kit (Randox Laboratories, UK) and an automated analyzer (Olympus AU 560). The following reagents were used: reagent 1 (glutathione, glutathione reductase, and $\mathrm{NADPH}$ ) was prepared by dissolving one vial in $8 \mathrm{~mL}$ of respective buffer 2 . Reagent 2 was prepared freshly before use by dissolving in $52 \mathrm{~mL}$ of twice-distilled water with $10 \mu \mathrm{L}$ cumene hydroperoxide and by stirring the mixture at room temperature.

The dilution reagent for the samples was prepared by dissolving one vial in $200 \mathrm{~mL}$ twice-distilled water. The assay was calibrated according to the manufacturer's instructions using control Ransel GPX samples. The samples were diluted further with the dilution reagent initially at a 1:40 ratio and afterward at a 1:10 ratio before analysis. The automated analyzer processed the samples according to the previous assay parameters, giving a linear standard curve with $840 \mathrm{U} / \mathrm{L}$ maximum.

\section{MDA analysis}

Fresh liver samples were cut in small pieces and placed in Eppendorf vials to be frozen at $-80^{\circ} \mathrm{C}$ before analysis. The samples were defrosted and their wet weight measured before homogenization in $900 \mu \mathrm{L}$ phosphate-buffered saline containing $100 \mu \mathrm{L} 0.5 \%$ butylated hydroxytoluene (Aldrich, USA) prepared in ethanol. Homogenates $(25 \mu \mathrm{L})$ prepared this way were kept in Eppendorf vials at $-80^{\circ} \mathrm{C}$ until MDA analysis.

MDA standards were prepared with 1,1,3,3-tetraethoxypropane (Sigma) as $0,0.31,0.63,1.25,2.5,5$, and $10 \mu \mathrm{M}$ solutions. Briefly, $25-\mu \mathrm{L}$ samples were mixed with $225 \mu \mathrm{L}$ water, $375 \mu \mathrm{L} 0.44 \mathrm{mM} \mathrm{H}_{3} \mathrm{PO}_{4}$ (Kemika), and $125 \mu \mathrm{L} 42 \mathrm{mM}$ thiobarbituric acid (Sigma) and were boiled at $100^{\circ} \mathrm{C}$ for $60 \mathrm{~min}$ and then cooled on ice. Upon cooling, alkaline methanol was added to the samples in a 1:1 ratio. The samples were centrifuged for $20 \mathrm{~min}$ at $3619 \mathrm{~g}$, and supernatants were analyzed at once. MDA was determined using an HPLC System Gold 128 analyzer (Beckman, Germany) with Midas auto-sampler (Spark,
Holland) and Shimadzu RF-535 fluorescent detector (Shimadzu, Japan) with 527-nm excitation and 550-nm emission. The mobile phase consisted of $50 \mathrm{mM} \mathrm{KH}_{2} \mathrm{PO}_{4}$ (Kemika), pH 6.8, and 40\% (v/v) methanol (Merck, Germany), with a $1 \mathrm{~mL} / \mathrm{min}$ flow applied for $100-\mu \mathrm{L}$ samples analyzed on a Spherisorb column ODS2, $5 \mu \mathrm{m}$, $4.6 \times 150 \mathrm{~mm}$ (Walters, USA).

\section{Statistical analysis}

Analysis of variance (ANOVA) for repeated measurements was used to compare responses, as a function of dose at each sampling time, and their interactions. Where ANOVA demonstrated a significant difference, we ran the post hoc LSD test of multiple comparisons to accurately determine to which doses and groups this significant difference applied. Statistical significance was set at $\mathrm{P} \leq 0.05$ (Statistica 6.0, USA).

\section{Results}

\section{In vivo $\mathrm{MN}$ assay in pregnant dams}

Baseline MN frequency in dams on day 12 of pregnancy was $0.86 \pm 0.90$ per 1000 reticulocytes. CP induced the highest MN frequency $(8.50 \pm 1.70 \mathrm{MN}$ per 1000 reticulocytes) $48 \mathrm{~h}$ after administration. Significantly higher MN frequencies were observed $48 \mathrm{~h}$ after the administration of 5-nitrofurantoin and sodium valproate $(4.13 \pm 1.96$ and $5.17 \pm 1.17$ per 1000 reticulocytes, respectively; Table 2). Fluconazole and paracetamol did not increase the MN frequency. No significant difference was observed between the group receiving a combination of 5-nitrofurantoin and paracetamol and the groups treated with 5-nitrofurantoin or paracetamol alone.

\section{In vivo $\mathrm{MN}$ assay in pups}

In newborn pups transplacentally exposed to $\mathrm{CP}, \mathrm{MN}$ frequency was $2.40 \pm 1.34$ per 1000 reticulocytes. In newborn pups of dams exposed to physiological saline, the $\mathrm{MN}$ frequency was $1.67 \pm 1.03$ per 1000 reticulocytes. All drugs tested increased the MN frequency in newborn pups. MN frequencies for each compound tested are shown in Table 2. The highest MN frequency in pups was caused by fluconazole (5.58 \pm 0.79 per 1000 reticulocytes). 
Table 2. Micronucleus frequency in pregnant mice and newborn pups (6-18 $\mathrm{h}$ after delivery), transplacentally exposed to the drugs tested and physiological saline.

\begin{tabular}{lccc}
\hline Drug & \multicolumn{2}{c}{ Pregnant mice } & Pups \\
\cline { 2 - 3 } & $0 \mathrm{~h}$ & $48 \mathrm{~h}$ & $6-18 \mathrm{~h}$ \\
\hline Negative control & $0.86 \pm 0.90$ & $1.00 \pm 0.58$ & $1.67 \pm 1.03$ \\
Positive control & $1.38 \pm 0.74$ & $8.50 \pm 1.70^{*}$ & $2.40 \pm 1.34$ \\
5-Nitrofurantoin & $1.13 \pm 0.83$ & $4.13 \pm 1.96^{*}$ & $5.00 \pm 1.00^{*}$ \\
Fluconazole & $1.44 \pm 0.73$ & $2.11 \pm 1.45$ & $5.58 \pm 0.79^{*}$ \\
Paracetamol & $1.00 \pm 0.76$ & $3.25 \pm 2.19$ & $3.80 \pm 1.10^{*}$ \\
5-Nitrofurantoin + paracetamol & $1.00 \pm 0.76$ & $4.50 \pm 1.07^{*}$ & $5.00 \pm 0.63^{*}$ \\
Sodium valproate & $1.33 \pm 0.52$ & $5.17 \pm 1.17^{*}$ & $5.20 \pm 1.48^{*}$ \\
\hline
\end{tabular}

Data are reported as means \pm SD per 1000 reticulocytes. Negative control: physiological saline; positive control: cyclophosphamide. ${ }^{*} \mathrm{P} \leq 0.05$ compared to baseline values (ANOVA).

No significant difference was observed between the group receiving the combination of 5-nitrofurantoin and paracetamol and the groups treated with 5-nitrofurantoin or paracetamol alone. Not a single animal showed malformations and no stillborns were detected.

\section{Biochemical analysis}

GPX. GPX activity in the hemolysate of newborn pups ranged from 1588.33 to $2561.67 \mathrm{U} / \mathrm{L}$. In the saline control group, it was $2134.29 \mathrm{U} / \mathrm{L}$. Only the paracetamol and 5nitrofurantoin + paracetamol groups were significantly different $(P \leq 0.05)$ from the control (Table 3$)$.

MDA. Liver MDA concentrations in newborn pups ranged from 83.71 to $233.83 \mathrm{pmol} / \mathrm{kg}$. All groups showed a significant difference $(P<0.05)$ from the saline control (233.83 pmol/kg). Group 6 (5-nitrofurantoin + paracetamol) did not differ significantly from Group 3 (5-nitrofurantoin alone) or Group 5 (paracetamol alone; Table 3).

\section{Discussion}

There are many gaps in the knowledge about metabolic disturbances and genome damage in fetuses of women taking drugs during pregnancy. Any linear correlation of the biological effects of transplacental drugs and health risks with the adult population would disregard specific biological and pharmacological interactions pertinent to fetal development. A causal relationship between genotoxic agents and increased risk of cancer has been evidenced by animal and epidemiological studies (32). In order to elucidate the transplacental effects of drugs commonly prescribed during pregnancy, we used an animal model to estimate the genome damage in pregnant mice and their pups. Additionally, genome damage, measured in vivo with the $\mathrm{MN}$ assay, was compared with detoxification capacity and the level of lipid peroxidation in newborn mice. This is also the first comprehensive study of GPX and MDA levels in mice exposed to xenobiotics during fetal development, and provides referral control values for the respective parameters of major antioxidant metabolism and lipid peroxidation in mice.

Whyatt et al. (33) have shown that glutathione transferase activity is critical for interpretation of DNA damage in maternal and newborn white blood cells after exposure to urban pollution. Low glutathione levels and

Table 3. Glutathione peroxidase (GPX) and malondialdehyde (MDA) levels (6-18 h after delivery) of the newborn mice transplacentally exposed to the drugs tested or saline.

\begin{tabular}{llcc}
\hline Group & \multicolumn{1}{c}{ Drug } & GPX $(\mathrm{U} / \mathrm{L})$ & MDA $(\mathrm{pmol} / \mathrm{kg})$ \\
\hline 1 & Negative control & $2134.29 \pm 320.36$ & $233.83 \pm 79.90$ \\
2 & Positive control & $2336.00 \pm 277.27$ & $107.60 \pm 19.01^{*}$ \\
3 & 5-Nitrofurantoin & $2296.25 \pm 488.46$ & $112.38 \pm 44.04^{*}$ \\
4 & Fluconazole & $2198.33 \pm 576.73$ & $146.71 \pm 36.25^{*}$ \\
5 & Paracetamol & $1588.33 \pm 408.58^{*}$ & $121.50 \pm 36.42^{*}$ \\
6 & 5-Nitrofurantoin + paracetamol & $1667.14 \pm 410.15^{*}$ & $83.71 \pm 41.54^{*}$ \\
7 & Sodium valproate & $2561.67 \pm 552.36$ & $96.00 \pm 54.93^{*}$ \\
\hline
\end{tabular}

Data are reported as means $\pm S D$. Negative control: physiological saline; positive control: cyclophosphamide. * $P \leq 0.05$ compared to saline control for GPX and MDA (post hoc LSD test). 
high glutathione disulfide/glutathione ratios have been associated with various illnesses (34). Tissue-specific and developmental variations in glutathione S-transferase (GST) mRNA expression were demonstrated in human prenatal liver, where total GPX and GST-dependent peroxidase activities were 9 to 18 times lower than in the adult liver (34).

Because the major part of fetal hematopoiesis occurs in the liver, we believe that the data obtained from peripheral blood hemolysate in our model are related to the onset of oxidative stress in the liver tissue. As shown by Gehringer et al. (35), a single dose of hepatotoxic compounds causes a significant increase in liver lipid peroxidation in the first $12 \mathrm{~h}$, after which it drops until the third day after treatment, while GPX activity increases significantly at the same time. This is in favor of the glutathione system participation, including GPX, whose synthesis is increased as the result of enhanced transcription of the enzyme and, consequently, increased attenuation of lipid peroxidation.

In the present study, newborn pups were killed not later than $18 \mathrm{~h}$ after birth, which is about 8 days after the last drug administration. Such timing enables analysis during the late phase of defense against hepatotoxicity, which means that the phase of increased lipid peroxidation in the liver has already ended. What follows is the increased transcription of enzymes GPX and gammaglutamyl transpeptidase, which inhibit glutathione synthesis in the liver. In the current study, only transplacental exposure of newborn pups to paracetamol and combined exposure to paracetamol and 5-nitrofurantoin significantly decreased levels of GPX. All compounds tested significantly decreased MDA levels, but the most pronounced effect was detected in the case of paracetamol and combined exposure to paracematol and 5-nitrofurantoin. Excessive doses of paracetamol deplete glutathione reserves, and the reactive paracetamol metabolite $\mathrm{N}$ acetyl-p-benzoquinoneimine easily binds to proteins and DNA, causing significant liver damage (36). According to our results, no synergy or antagonism can be detected by applied methods for combined transplacental exposure to paracetamol and 5-nitrofurantoin by the methods used. Therefore, the reduction of GPX reflected systemic oxidative stress, which is known to occur with the paracetamol treatment, while the reduction of MDA in the liver can be interpreted as an unspecific reaction to the various drug treatments that might have cytotoxic, and in particular hepatotoxic, effects associated with oxidative stress and lipid peroxidation. Therefore, of additional value would be immunohistochemical analyses of different organs and tissues for MDA and other biomarkers of lipid peroxidation, in particular HNE. HNE is known to act as a bifunctional, concentration- and cytokine-dependent growth regulator that might also affect glutathione metabolism and trigger hormetic antioxidant responses to severe stress (37), which might eventually be involved in consequential lowering of liver MDA, as was observed in the study. It is worth mentioning also that placental HNE was found recently to be related to reduced fetal growth rate in humans (38), but such analyses could not have been done in the current study, due to technical limitations of the immunohistochemical method based on mice monoclonal antibodies (39). However, in future studies we will try to extend the present study also in this direction and evaluate the possible involvement of HNE in the toxicity of drugs crossing the transplacental barrier.

This is the first study of genome damage in pups caused by transplacental exposure during organogenesis using an in vivo MN assay. The advantage of a small sample size required by this method additionally enabled the comparison of genome damage in pregnant dams and their own pups, unlike other genotoxicological methods where the sample size necessitates the killing of animals or may impact the outcome of pregnancy. Results showed that sodium valproate and 5-nitrofurantoin significantly increased $\mathrm{MN}$ frequency in pregnant dams compared to controls, unlike fluconazole and paracetamol. The absence of an increased MN frequency in dams after exposure to fluconazole and paracetamol and increased MN frequency in their pups confirmed that genome damage in the mother cannot be used as a predictor of genome damage in newborns. This was especially evident for fluconazole, which caused the highest MN frequency in pups. A common combined application of therapy by antibiotics and antipyretics, such as that of 5nitrofurantoin and paracetamol, significantly increased $\mathrm{MN}$ frequency in pregnant dams but caused no significant difference in MN frequency between exposure to 5nitrofurantoin and the combined effect of 5-nitrofurantoin and paracetamol.

In newborn pups, the MN frequency was significantly higher for all of the compounds tested compared to the negative control. This shows that all of the compounds tested passed the placental barrier and caused genotoxic effects in newborn pups. These findings are in accordance with previous reports that showed that fluconazole and 5-nitrofurantoin also significantly increased $\mathrm{MN}$ frequency in prepubertal animals $(12,17)$. The combined effect of 5-nitrofurantoin and paracetamol in pups, the same as in dams, showed a significantly increased frequency of $\mathrm{MN}$, but there was no significant difference in $\mathrm{MN}$ frequency between exposure to 5-nitrofurantoin and the combined effect of 5-nitrofurantoin and paracetamol. It could be suggested that the genome damage caused by these compounds alone or combined follow the same mechanisms and that these mechanisms are not age related.

The comparison of the MN frequency in pups with GPX and MDA did not show a correlation, except for the synergistic action of 5-nitrofurantoin and paracetamol, which caused both increased $M N$ frequency and the lowest MDA level among the compounds tested. The 
described synergisms revealed significant side effects in the case of combined therapy, with possible health risks that should be investigated in the future.

Both the observed difference in susceptibility to xenobiotics between pregnant dams and newborns and

\section{References}

1. Mongraw-Chaffin ML, Cohn BA, Anglemyer AT, Cohen RD, Christianson RE. Maternal smoking, alcohol, and coffee use during pregnancy and son's risk of testicular cancer. Alcohol 2009; 43: 241-245, doi: 10.1016/j.alcohol.2008.12.002.

2. Jeong $\mathrm{H}$. Altered drug metabolism during pregnancy: hormonal regulation of drug-metabolizing enzymes. Expert Opin Drug Metab Toxicol 2010; 6: 689-699, doi: 10.1517| 17425251003677755.

3. Slupphaug G, Kavli B, Krokan HE. The interacting pathways for prevention and repair of oxidative DNA damage. Mutat Res 2003; 531: 231-251, doi: 10.1016/j.mrfmmm.2003.06. 002.

4. Blair IA. DNA adducts with lipid peroxidation products. $J$ Biol Chem 2008; 283: 15545-15549, doi: 10.1074/jbc. R700051200.

5. Esterbauer H, Eckl P, Ortner A. Possible mutagens derived from lipids and lipid precursors. Mutat Res 1990; 238: 223233, doi: 10.1016/0165-1110(90)90014-3.

6. Gueraud F, Atalay M, Bresgen N, Cipak A, Eckl PM, Huc L, et al. Chemistry and biochemistry of lipid peroxidation products. Free Radic Res 2010; 44: 1098-1124, doi: 10. 3109/10715762.2010.498477.

7. Fowden AL, Giussani DA, Forhead AJ. Intrauterine programming of physiological systems: causes and consequences. Physiology 2006; 21: 29-37, doi: 10.1152/physiol. 00050.2005

8. Klinger W. Developmental pharmacology and toxicology: biotransformation of drugs and other xenobiotics during postnatal development. Eur J Drug Metab Pharmacokinet 2005; 30: 3-17, doi: 10.1007/BF03226403.

9. Elbarbry F, Alcorn J. Ontogeny of glutathione and glutathione-related antioxidant enzymes in rat liver. Res Vet Sci 2009; 87: 242-244, doi: 10.1016/j.rvsc.2009.03.004.

10. Dietert RR, Piepenbrink MS. Perinatal immunotoxicity: why adult exposure assessment fails to predict risk. Environ Health Perspect 2006; 114: 477-483, doi: 10.1289/ehp. 8566.

11. Neto JA, Oliveira-Filho RM, Simoes MJ, Soares JM Jr, Kulay L Jr. Long-term acetaminophen (paracetamol) treatment causes liver and kidney ultra-structural changes during rat pregnancy. Clin Exp Obstet Gynecol 2004; 31: 221-224.

12. Fucic A, Markovic D, Ferencic Z, Mildner B, Jazbec AM, Spoljar JB. Comparison of genomic damage caused by 5 nitrofurantoin in young and adult mice using the in vivo micronucleus assay. Environ Mol Mutagen 2005; 46: 59-63, doi: 10.1002/em.20129.

13. Crider KS, Cleves MA, Reefhuis J, Berry RJ, Hobbs CA, Hu DJ. Antibacterial medication use during pregnancy and risk of birth defects: National Birth Defects Prevention Study. Arch Pediatr Adolesc Med 2009; 163: 978-985, doi: 10.1001/archpediatrics.2009.188

14. Graf WD, Oleinik OE, Glauser TA, Maertens P, Eder DN, the unsuccessful elimination of genome damage in developing organisms exposed in utero, lipid peroxidation, and metabolic disturbances measurable in newborns confirm their specific vulnerability and show the need for further investigation of these processes.

Pippenger CE. Altered antioxidant enzyme activities in children with a serious adverse experience related to valproic acid therapy. Neuropediatrics 1998; 29: 195-201, doi: 10.1055/s-2007-973560.

15. Al Deeb S, Al Moutaery K, Arshaduddin M, Tariq M. Vitamin $E$ decreases valproic acid induced neural tube defects in mice. Neurosci Lett 2000; 292: 179-182, doi: 10.1016/ S0304-3940(00)01457-9.

16. Norgaard M, Pedersen L, Gislum M, Erichsen R, Sogaard $\mathrm{KK}$, Schonheyder HC, et al. Maternal use of fluconazole and risk of congenital malformations: a Danish population-based cohort study. J Antimicrob Chemother 2008; 62: 172-176, doi: $10.1093 / j a c / d k n 157$.

17. Fucic A, Markovic D, Herceg Z, Gamulin M, Katic J, Stojkovic R, et al. Developmental and transplacental genotoxicology: fluconazole. Mutat Res 2008; 657: 43-47, doi: 10.1016/j.mrgentox.2008.08.008.

18. Marotta F, Tiboni GM. Molecular aspects of azoles-induced teratogenesis. Expert Opin Drug Metab Toxicol 2010; 6: 461-482, doi: 10.1517/17425251003592111.

19. Le J, Briggs GG, McKeown A, Bustillo G. Urinary tract infections during pregnancy. Ann Pharmacother 2004; 38: 1692-1701, doi: 10.1345/aph.1D630.

20. Mendling W, Brasch J. Guideline vulvovaginal candidosis (2010) of the German Society for Gynecology and Obstetrics, the Working Group for Infections and Infectimmunology in Gynecology and Obstetrics, the German Society of Dermatology, the Board of German Dermatologists and the German Speaking Mycological Society. Mycoses 2012; 55 (Suppl 3): 1-13, doi: 10.1111/j.1439-0507.2012.02185.x.

21. Hernandez-Diaz S, Smith CR, Shen A, Mittendorf R, Hauser WA, Yerby $M$, et al. Comparative safety of antiepileptic drugs during pregnancy. Neurology 2012; 78: 1692-1699, doi: 10.1212/WNL.0b013e3182574f39.

22. Andersen AB, Farkas DK, Mehnert F, Ehrenstein V, Erichsen R. Use of prescription paracetamol during pregnancy and risk of asthma in children: a population-based Danish cohort study. Clin Epidemiol 2012; 4: 33-40.

23. Hayashi M, Morita T, Kodama $Y$, Sofuni T, Ishidate M Jr. The micronucleus assay with mouse peripheral blood reticulocytes using acridine orange-coated slides. Mutat Res 1990; 245: 245-249, doi: 10.1016/0165-7992(90) 90153-B.

24. Levy G, Garrettson LK, Soda DM. Letter: Evidence of placental transfer of acetaminophen. Pediatrics 1975; 55: 895.

25. Aleck KA, Bartley DL. Multiple malformation syndrome following fluconazole use in pregnancy: report of an additional patient. Am J Med Genet 1997; 72: 253-256, doi: 10.1002/(SICI)1096-8628(19971031)72:3<253::AIDAJMG1>3.0.CO;2-S.

26. Lopez-Rangel E, Van Allen MI. Prenatal exposure to 
fluconazole: an identifiable dysmorphic phenotype. Birth Defects Res A Clin Mol Teratol 2005; 73: 919-923, doi: 10.1002/bdra.20189.

27. De Santis M, De Luca C, Mappa I, Cesari E, Quattrocchi T, Spagnuolo T, et al. Antiepileptic drugs during pregnancy: pharmacokinetics and transplacental transfer. Curr Pharm Biotechnol 2011; 12: 781-788, doi: 10.2174/13892011179 5470958.

28. Zemlickis D, Lishner M, Erlich R, Koren G. Teratogenicity and carcinogenicity in a twin exposed in utero to cyclophosphamide. Teratog Carcinog Mutagen 1993; 13: 139-143, doi: 10.1002/tcm.1770130304.

29. D'Arcy PF. Nitrofurantoin. Drug Intell Clin Pharm 1985; 19: 540-547.

30. U.S. Department of Health and Human Services Food and Drug Administration Center for Drug Evaluation and Research (CDER). Guidance for Industry, Estimating the maximum safe starting dose in initial clinical trial for therapeutics an adult healthy volunteer. Silver Spring: FDA; 2005.

31. Kaufman $\mathrm{MH}$, Bard JBL. The anatomical basis of mouse development. San Diego: Academic Press; 1999.

32. Norppa H, Bonassi S, Hansteen IL, Hagmar L, Stromberg U, Rossner $\mathrm{P}$, et al. Chromosomal aberrations and SCEs as biomarkers of cancer risk. Mutat Res 2006; 600: 37-45, doi: 10.1016/j.mrfmmm.2006.05.030.

33. Whyatt RM, Perera FP, Jedrychowski W, Santella RM, Garte S, Bell DA. Association between polycyclic aromatic hydrocarbon-DNA adduct levels in maternal and newborn white blood cells and glutathione S-transferase P1 and CYP1A1 polymorphisms. Cancer Epidemiol Biomarkers Prev 2000; 9: 207-212.

34. Mills BJ, Weiss MM, Lang CA, Liu MC, Ziegler C. Blood glutathione and cysteine changes in cardiovascular disease. J Lab Clin Med 2000; 135: 396-401, doi: 10.1067/mlc.2000. 105976.

35. Gehringer MM, Shephard EG, Downing TG, Wiegand C, Neilan BA. An investigation into the detoxification of microcystin-LR by the glutathione pathway in Balb/c mice. Int J Biochem Cell Biol 2004; 36: 931-941, doi: 10.1016/ j.biocel.2003.10.012.

36. Rogers LK, Moorthy B, Smith CV. Acetaminophen binds to mouse hepatic and renal DNA at human therapeutic doses. Chem Res Toxicol 1997; 10: 470-476, doi: 10.1021/ tx960159i.

37. Zarkovic N. 4-Hydroxynonenal as a bioactive marker of pathophysiological processes. Mol Aspects Med 2003; 24: 281-291, doi: 10.1016/S0098-2997(03)00023-2.

38. Gveric-Ahmetasevic S, Sunjic SB, Skala H, Andrisic L, Stroser M, Zarkovic K, et al. Oxidative stress in small-forgestational age (SGA) term newborns and their mothers. Free Radic Res 2009; 43: 376-384, doi: 10.1080/1071576 0902783285

39. Spickett CM, Wiswedel I, Siems W, Zarkovic K, Zarkovic N. Advances in methods for the determination of biologically relevant lipid peroxidation products. Free Radic Res 2010; 44: 1172-1202, doi: 10.3109/10715762.2010.498476. 